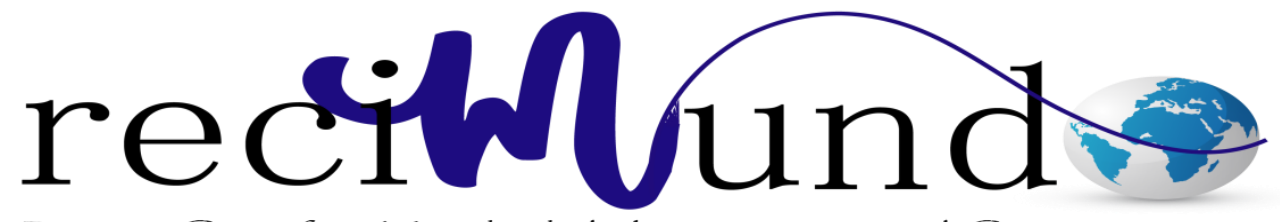

Revista Científica Mundo de la Investigación y el Conocimiento

Marjoire Catalina Valle Espin ${ }^{\text {a }}$; Freddy Eduardo Villacreces Garcia ${ }^{\text {b; Julio }}$ Guillermo Chippe Villacres ${ }^{\text {c; }}$ Maria Auxiliadora Palma Roditi ${ }^{\text {d }}$ Karina Jessenia Jarrin Maisincho ${ }^{\mathrm{e}}$

Factores que influyen en la no adherencia al tratamiento antirretroviral en pacientes con VIH/ Sida

Revista Científica Mundo de la Investigación y el Conocimiento. Vol. 2 núm., 1, febrero, ISSN: 2588-073X, 2018, pp. 488-501

DOI: 10.26820/recimundo/2.1.2018.488-501

Editorial Saberes del Conocimiento
a. kattyvalle@yahoo.com
b. freddyvillacres@gmail.com
c. igchippe@gmail.com
d. mariupalma@hotmail.com
e. drakarinajarrinmed@hotmail.com 


\section{Factores que influyen en la no adherencia al tratamiento antirretroviral en pacientes con VIH/ Sida}

Vol. 2, núm. 1., (2018)

Marjoire Catalina Valle Espin; Freddy Eduardo Villacreces Garcia; Julio Guillermo Chippe

Villacres; Maria Auxiliadora Palma Roditi; Karina Jessenia Jarrin Maisincho

\section{RESUMEN}

Objetivo: Identificar factores que influyen en la no adherencia terapéutica con antiretrovirales (ARV) en pacientes con VIH/SIDA y que desfavorecen el estado clínico y el pronóstico de los pacientes infectados Métodos: estudio cualitativo-cuantitativo descriptivo en pacientes que identifica aspectos socio afectivos que inciden en la no adherencia en el hospital "Martín Icaza" en la ciudad de Babahoyo - Los Ríos Ecuador. Se aplicó un cuestionario de datos sociodemográficos, Aspectos Psico-sociales y calidad de vida Resultados: se clasificó a las pacientes en dos grupos, el Grupo 1 Adherente y grupo 2 no adherente. Se confirman los resultados con CD4 y carga viral según los resultados, tomando como referencia cifras de CD4 por arriba de $200 \mathrm{cel} / \mathrm{mm} 3$ y el grupo 2 incluye cifras por debajo a $200 \mathrm{cel} / \mathrm{mm} 3$ y carga viral en relación a el primer grupo cifras menores a $50 \mathrm{copias} / \mathrm{ml}$ siendo estos valores indetectables y el grupo 2 mayor o igual a 50 copias $/ \mathrm{ml}$ detectables y se obtuvieron los resultados con la muestra poblacional de 85 pacientes de las cuales el $45 \%$ pertenecieron a la categoría de no adherentes de esta misma población estudiada se encasillaron una mayoría con falta de recursos económicos para cumplir con la terapia ARV y controles médicos, nivel bajo de autoestima, maltrato físico y el $90 \%$ se sintieron discriminadas. Discusión la adherencia, con enfoque cuantitativo da valores significativos para medir magnitud y no causa, relacionan la adherencia con la evolución clínica pero los estudios se quedan en examinar los niveles y porcentajes de la adherencia sin revisar el porqué de la no adherencia. Está en discusión, considerar a más de los referentes cuantitativos, aspectos como cultura y estilos de vida con enfoque cualitativo. Conclusiones existe relación entre adhesión al tratamiento y variables psicológicas y sociales. Los niveles de afectación la autoestima y el apoyo familiar así son claves en la adherencia. La calidad de vida y factores sociales inciden en que, el $45 \%$ de pacientes no cumplan con los parámetros de adherencia y la mala adherencia es causa de enfermedades oportunistas. La "feminización" se pone en evidencia, hace una década había 10 hombres con VIH por cada mujer con VIH, en 2005 hubo 2,4 hombres por cada mujer, y en lo que va del 2016 son apenas 1,7 hombres por cada mujer. Es decir, hay cada vez más mujeres afectadas por el VIH. La feminización rompe el mito que la enfermedad es exclusiva de prostitutas, la mayoría de las mujeres a infectadas es de ocupación que haceres domésticos.

Palabras clave: VIH SIDA, TARV, adherencia, factores psicosociales. 


\section{Factores que influyen en la no adherencia al tratamiento antirretroviral en pacientes con VIH/ Sida}

Vol. 2, núm. 1., (2018)

Marjoire Catalina Valle Espin; Freddy Eduardo Villacreces Garcia; Julio Guillermo Chippe Villacres; Maria Auxiliadora Palma Roditi; Karina Jessenia Jarrin Maisincho

\section{ABSTRACT}

Objective: Identify factors that influence the therapeutic non-adherence with antiretroviral (ARV) in patients with HIV/AIDS and that the clinical condition and the prognosis of patients infected disadvantage methods: descriptive quantitative study in patients that identifies affective social aspects affecting the non-adherence in the "Martín Icaza" hospital in the city of Babahoyo - Los Ríos Ecuador. A questionnaire of socio-demographic data, psycho-social aspects and quality of life results: classified patients into two groups, the adherent Group 1 and group 2 nonsticks. Confirmed the results with CD4 and viral according he load, with reference to numbers of CD4 above $200 \mathrm{cel} / \mathrm{mm} 3$ and group 2 includes numbers below $200 \mathrm{cel} / \mathrm{mm} 3$ and load in relation to the first group numbers less than 50 copies/ml being undetectable values and group 2 greater than or equal to 50 copies/ml detectable and the results, with the population sample of 85 patients of which $45 \%$ belonged to the category of non-adherent of this same population studied is pigeonholed a majority with lack of economic resources to comply with ARV therapy and medical controls, low level of self-esteem, physical abuse and 90\% felt discriminated against. Discussion adhesion, with quantitative approach gives significant values to measure the magnitude and not the cause, they relate the adhesion with the clinical evolution but studies thus raised are to examine the levels and rates of adherence without reviewing the because of nonadherence. It is in discussion, consider more .conclusions there is a relationship between adherence to treatment and psychological and social variables. Levels of involvement to the selfesteem and family support so are key in adhesion. The quality of life and social factors affect that $45 \%$ of patients do not meet the parameters of adhesion and the poor adhesion is cause of opportunistic diseases. Feminization" is put in evidence a decade ago had 10 men with HIV for every woman with HIV, in 2005 there were 2.4 men for every woman, and so far the 2016 are just 1.7 men for every woman. I.e., there are more and more women affected by HIV. Feminization breaks the myth that the disease is exclusive of prostitutes, the majority of women infected is occupation than domestic doings.

Keywords: HIV AIDS, art, adhesion, psychosocial factors. 


\section{Factores que influyen en la no adherencia al tratamiento antirretroviral en pacientes con VIH/ Sida}

Vol. 2, núm. 1., (2018)

Marjoire Catalina Valle Espin; Freddy Eduardo Villacreces Garcia; Julio Guillermo Chippe Villacres; Maria Auxiliadora Palma Roditi; Karina Jessenia Jarrin Maisincho

\section{Introducción.}

Las personas que padecen VIH/SIDA requieren de adaptación al tratamiento ARV. Los estudios de adherencia se efectúan desde exploraciones basadas en modelos teóricos para evaluar factores condicionantes y variables predictores del tratamiento al paciente con VIH/Sida. El tema de la adherencia a TARV involucra aspectos no solamente de carácter descriptivo y al examinar la conducta humana la perspectiva de la investigación se introduce en el plano de las subjetividades y prejuicios que se observan desde la sociedad en sus modelos mentales, afectando el entorno que los rodea. $(1,2)$

La inequidad, las asimetrías de género, la vulnerabilidad social, la discriminación, son aspectos políticos y socioculturales, que repercuten en el estado clínico y emocional de aspectos políticos y socioculturales, que repercuten en el estado clínico y emocional de los pacientes. Mellins, Kang, Cheng-Shium, Havens y Chesney; 2013 determinaron que existía una alta tasa de trastornos emocionales como depresión en una tasa porcentual del 50 y $89 \%$. Otros estudios también han mostrado una alta incidencia de depresión en los pacientes con VIH/SIDA, sobre todo en pacientes mujeres. (3) Jones e. 2013; Wagner y Ghosh-Dastidar, 2013, Kimmerlin. 2010; encontraron relevante prevalencia de síndromes depresivos además de otros factores psicosociales como son la ansiedad, el estrés y el apoyo social e incluye un grupo de características que actúan para definirla como: la zona de residencia, el estrato socio-económico, la educación, el tipo de afiliación al sistema de salud, el trabajo o las "formas de ganarse la vida", el nivel de ingresos y el acceso a recursos económicos como la propiedad y el crédito. (4) 


\section{Factores que influyen en la no adherencia al tratamiento antirretroviral en pacientes con VIH/ Sida}

Vol. 2, núm. 1., (2018)

Marjoire Catalina Valle Espin; Freddy Eduardo Villacreces Garcia; Julio Guillermo Chippe Villacres; Maria Auxiliadora Palma Roditi; Karina Jessenia Jarrin Maisincho

La búsqueda de no adherencia a la TARV en pacientes con VIH/SIDA Manejados en la Clínica de HIV del hospital Martín Icaza fenómeno de causa multifactorial permitió generar recomendaciones de manejo sostenible a pacientes afectados.

Las conclusiones de esta investigación, contribuirá a la reducción de enfermedades oportunistas producto de la baja carga inmunológica de los pacientes con abandono de TAR porque como lo establecen Avery, 2000; Borucki, 2000; las fallas de cumplimiento derivan de inmediato en infecciones oportunistas. (5)

Sobre la adherencia a los ARV y la situación de género en la mujer portadora del VIH/SIDA pocos han sido los estudios que evalúen las consecuencias con diagnóstico de la enfermedad. El diagnostico puede afectar a la mujer porque a la inequidad social se suma la inequidad de género, que agrega mayor vulnerabilidad a la mujer portadora. La población femenina afectada puede ser más impactada en su estado clínico y emocional como consecuencia de la infección y este factor afecta la esfera emocional en especial la autoestima que es un elemento clave en la adherencia.

Mellins, Kang, y colaboradores 2013; también determinaron la existencia de trastornos mentales en población de mujeres con VIH: encontrando síntomas depresivos en mujeres portadoras del VIH/SIDA además de otros factores. (3)

Un estudio realizado en Colombia país vecino a Ecuador con similitudes et no sociales en una muestra con 47 mujeres concluyo que el 23,4\% presenta indicadores clínicos de ansiedad, el 


\section{Factores que influyen en la no adherencia al tratamiento antirretroviral en pacientes con VIH/ Sida}

Vol. 2, núm. 1., (2018)

Marjoire Catalina Valle Espin; Freddy Eduardo Villacreces Garcia; Julio Guillermo Chippe Villacres; Maria Auxiliadora Palma Roditi; Karina Jessenia Jarrin Maisincho

10,63\% de depresión, el 6,38\% muestra bajos niveles de autoestima y el 100\% de las participantes variables de ansiedad y depresión. (6)

La condición de la mujer ecuatoriana como cualquier mujer latina, la conlleva a temores y perjuicios dentro de los cuales se involucra la estigmatización por parte del entorno social y familiar.

El objetivo del presente estudio es objetivo identificar factores sociales determinantes en la adherencia terapéutica con antiretrovirales con VIH/ SIDA esta relación ha sido tratada por la literatura científica con resultados diferentes, habitualmente, no se analizan los aspectos literatura científica con resultados diferentes, habitualmente, no se analizan los aspectos sociales como un grupo de condiciones que actúan conjuntamente para determinar, barreras y condiciones de vida que se asocian con su adherencia terapéutica.

\section{Metodología.}

Estudio cualitativo-cuantitativo descriptivo realizado en el periodo junio a diciembre del año 2016 con la participación de 85 pacientes con edades comprendidas entre 20 y 50 años de la provincia de Los Ríos en la clínica del VIH del Hospital Martin Icaza. Se aplicó cuestionario de datos socio-demográficos, Aspectos Psico-sociales, calidad de vida. Se complementó con entrevistas a usuarios y se coordinó con trabajo social y psicología el análisis de discurso de los pacientes para hacer objetiva la observación de conductas Las entrevistas se estructuraron sobre temas de: adherencia terapéutica, Calidad de vida, estigmatización y discriminación.

\section{Resultados.}




\section{Factores que influyen en la no adherencia al tratamiento antirretroviral en pacientes con VIH/ Sida}

Vol. 2, núm. 1., (2018)

Marjoire Catalina Valle Espin; Freddy Eduardo Villacreces Garcia; Julio Guillermo Chippe Villacres; Maria Auxiliadora Palma Roditi; Karina Jessenia Jarrin Maisincho

Encuesta realizada a 85 pacientes con diagnóstico de VIH/SIDA edades comprendidas entre 20 a 45 años presento los siguientes resultados.

\begin{tabular}{|l|c|r|}
\hline Características Socio-Demográficas & $\mathbf{N}^{\circ}$ & $\%$ \\
\hline Procedencia & 52 & 61 \\
\hline Los Ríos & 33 & 39 \\
\hline Otras provincias & 10 & 12 \\
\hline Estado Civil & 12 & 14 \\
\hline Solteros & 60 & 71 \\
\hline Unión libre & 3 & 4 \\
\hline divorcio & \multicolumn{3}{|l|}{} \\
\hline Viuda & 29 & 34 \\
\hline Edad & 36 & 42 \\
\hline 20-30 años & 20 & 24 \\
\hline 31-40 años & \multicolumn{3}{|c|}{85} \\
\hline 41-50 años
\end{tabular}

Tabla 1. Características socio-demográficas de los pacientes

masculino femenino total

$\begin{array}{lll}52 & 33 & 85\end{array}$

\section{Cuadro $N^{\circ} 1 .-$ distribución de género y ocupación}

A pesar de que la mayor prevalencia de VIH se sitúa en población masculina en Ecuador y este estudio lo ratifica el fenómeno de la feminización es un factor a ser considerado tendencialmente pues es una de las áreas de más impacto por la transmisión vertical. Al igual que 


\section{Factores que influyen en la no adherencia al tratamiento antirretroviral en pacientes con VIH/ Sida}

Vol. 2, núm. 1., (2018)

Marjoire Catalina Valle Espin; Freddy Eduardo Villacreces Garcia; Julio Guillermo Chippe Villacres; Maria Auxiliadora Palma Roditi; Karina Jessenia Jarrin Maisincho

toda la nación el tamizaje de las madres en gestación está bien encaminada y su porcentaje de gestación llega ya al 90\% (MSP).

La "feminización" de la epidemia es continúa, hace una década había 10 hombres con VIH por cada mujer con VIH, en 2005 hubo 2,4 hombres por cada mujer, y en lo que va del 2016 son apenas 1,7 hombres por cada mujer. Es decir, hay cada vez más mujeres afectadas por el VIH.

La feminización rompe el mito que la enfermedad es exclusiva de prostitutas, la mayoría de las mujeres a infectadas es de ocupación que haceres domésticos.

\begin{tabular}{|c|c|}
\hline $\begin{array}{l}\text { Trabajadoras del } \\
\text { hogar }\end{array}$ & $\begin{array}{c}\text { profesionales Trabajadoras } \\
\text { sexuales }\end{array}$ \\
\hline 18 & 10 \\
\hline
\end{tabular}

\section{Cuadro $N^{\circ}$ 2.- Situación laboral}

La Conferencia Internacional del Trabajo (CIT) de junio de 2009, en su Resolución sobre a la igualdad de género identificó al VIH como uno de los mayores retos para la consecución de la igualdad de género. Siendo la mayoría de mujeres de ocupación en que haceres del hogar, el que ha sido examinados en este estudio, es muy probable que esta situación esconda una realidad de discriminación en el mundo laboral formal. 


\section{Factores que influyen en la no adherencia al tratamiento antirretroviral en}

pacientes con VIH/ Sida

Vol. 2, núm. 1., (2018)

Marjoire Catalina Valle Espin; Freddy Eduardo Villacreces Garcia; Julio Guillermo Chippe Villacres; Maria Auxiliadora Palma Roditi; Karina Jessenia Jarrin Maisincho

\begin{tabular}{|l|l|c|}
\hline \multicolumn{1}{|c|}{ Características } & N & $\%$ \\
\hline Ingreso económico & 31 & 37 \\
\hline Menor al básico & 19 & 22 \\
\hline Sueldo básico & 8 & 9 \\
\hline Superior al básico & 27 & 32 \\
\hline Ninguno & 68 & 80 \\
\hline Ocupación & \multicolumn{3}{|l|}{} \\
\hline Ama de casa & 2 & 2 \\
\hline Empleado público & 15 & 18 \\
\hline Empleado privado & \multicolumn{2}{|l|}{} \\
\hline Nivel instrucción & 58 & 68 \\
\hline Primaria & 25 & 29 \\
\hline Bachiller & 2 & 2 \\
\hline Superior & 52 & 61 \\
\hline Religión & 27 & 32 \\
\hline Católica & 6 & 7 \\
\hline Evangélica
\end{tabular}

\begin{tabular}{|l|c|}
\hline Total de Pacientes & 85 \\
\hline
\end{tabular}

Tabla 2. Características socio económico y cultural de los pacientes VIH/Sida

\begin{tabular}{|c|c|c|c|c|c|c|c|c|}
\hline \multirow[t]{2}{*}{ Ingresos económicos } & \multicolumn{2}{|c|}{ Siempre } & \multicolumn{2}{|c|}{$\begin{array}{c}\text { Algunas } \\
\text { Veces }\end{array}$} & \multicolumn{2}{|c|}{$\begin{array}{l}\text { Pocas } \\
\text { veces }\end{array}$} & \multicolumn{2}{|c|}{ Nunca } \\
\hline & $\mathbf{N}^{\circ}$ & $\%$ & $\mathrm{~N}^{\circ}$ & $\%$ & $\mathrm{~N}^{\circ}$ & $\%$ & $\mathrm{~N}^{\circ}$ & $\%$ \\
\hline $\begin{array}{l}\text { ¿Tiene dificultades } \\
\text { económicas para retirar } \\
\text { sus medicamentos }\end{array}$ & 39 & 46 & 27 & 32 & 15 & $18 \%$ & 3 & 4 \\
\hline Recibe ayuda económica & 19 & 22 & 19 & 23 & 40 & $47 \%$ & 7 & 8 \\
\hline $\begin{array}{l}\text { ¿Mantiene la carga de } \\
\text { responsabilidad económica en } \\
\text { su hogar? }\end{array}$ & 49 & 58 & 14 & 17 & 12 & $14 \%$ & 9 & 11 \\
\hline
\end{tabular}

Tabla 3. Preguntas relacionadas al factor económico 


\section{Factores que influyen en la no adherencia al tratamiento antirretroviral en pacientes con VIH/ Sida}

Vol. 2, núm. 1., (2018)

Marjoire Catalina Valle Espin; Freddy Eduardo Villacreces Garcia; Julio Guillermo Chippe

Villacres; Maria Auxiliadora Palma Roditi; Karina Jessenia Jarrin Maisincho

El 37\% no gana más del sueldo básico mientras el 32\% no percibe sueldo alguno. El 46\% tiene dificultades para movilizarse a retirar los ARV y a los controles por tener otras responsabilidades económicas de manutención representado por el 58\% de esta muestra.

\begin{tabular}{|c|c|c|c|c|c|c|c|c|}
\hline \multirow{2}{*}{$\begin{array}{c}\text { Apoyo y discriminación: } \\
\text { Entorno Social }\end{array}$} & \multicolumn{2}{|c|}{ Nunca } & \multicolumn{2}{|c|}{ Siempre } & \multicolumn{2}{|c|}{$\begin{array}{c}\text { Algunas } \\
\text { veces }\end{array}$} & \multicolumn{2}{|c|}{$\begin{array}{l}\text { Solo una } \\
\text { vez }\end{array}$} \\
\hline & $\mathrm{N}^{0}$ & $\%$ & $\mathrm{~N}^{0}$ & $\%$ & $\mathrm{~N}^{\circ}$ & $\%$ & $\mathbf{N}^{\circ}$ & $\%$ \\
\hline $\begin{array}{l}\text { Cursa formas de Maltrato } \\
\text { psicológico? }\end{array}$ & 19 & 22 & 5 & 6 & 43 & 51 & 18 & 21 \\
\hline $\begin{array}{l}\text { Se ha sentido afectado } \\
\text { por agresión fisica? }\end{array}$ & 36 & 42 & 8 & 9 & 21 & 25 & 20 & 24 \\
\hline $\begin{array}{l}\text { Se ha sentido } \\
\text { discriminado de ser } \\
\text { portador VIH }\end{array}$ & 17 & 20 & 38 & 45 & 24 & 28 & 6 & 7 \\
\hline
\end{tabular}

\begin{tabular}{|l|c|}
\hline Total de Pacientes & 85 \\
\hline
\end{tabular}

Tabla 4. Estigma y discriminación de las pacientes con VIH/Sida

Respecto a estigma y discriminación casi la mitad de los pacientes estudiados 43 de 85 con el 51\%refirieron recibir maltrato psicológico en algunas ocasiones sin embargo el $42 \%$ refirió no haber recibido maltrato físico y ante la pregunta realizada ¿te sientes discriminado el 45\% manifestó sentirse discriminada el 28\% respondió que en algunas ocasiones se han sentido discriminadas, mientras el $20 \%$ manifestaron que nunca se han sentido así. 
Factores que influyen en la no adherencia al tratamiento antirretroviral en pacientes con VIH/ Sida

Vol. 2, núm. 1., (2018)

Marjoire Catalina Valle Espin; Freddy Eduardo Villacreces Garcia; Julio Guillermo Chippe Villacres; Maria Auxiliadora Palma Roditi; Karina Jessenia Jarrin Maisincho

\begin{tabular}{|c|c|c|c|c|c|c|c|c|}
\hline \multirow{2}{*}{$\begin{array}{l}\text { VALORACION } \\
\text { PSICOLOGICA }\end{array}$} & \multicolumn{2}{|c|}{ Nunca } & \multicolumn{2}{|c|}{ Siempr } & \multicolumn{2}{|c|}{$\begin{array}{c}\text { Algunas } \\
\text { veces }\end{array}$} & \multicolumn{2}{|c|}{$\begin{array}{l}\text { Pocas } \\
\text { veces }\end{array}$} \\
\hline & $\mathrm{N}^{\circ}$ & $\%$ & $\mathrm{~N}^{\circ}$ & $\%$ & $\mathrm{~N}^{\circ}$ & $\%$ & $\mathrm{~N}^{0}$ & $\%$ \\
\hline $\begin{array}{l}\text { ¿Se siente con la moral } \\
\text { decaído? }\end{array}$ & 16 & 19 & 21 & 25 & 28 & 33 & 20 & 23 \\
\hline ¿A menudo se siente triste? & 11 & 13 & 21 & 25 & 34 & 40 & 19 & 22 \\
\hline $\begin{array}{l}\text { ¿ha tenido ideas de } \\
\text { Suicidio? }\end{array}$ & 65 & 77 & 2 & 2 & 10 & 12 & 8 & 9 \\
\hline
\end{tabular}

\begin{tabular}{|l|c|}
\hline Total de Pacientes & 85 \\
\hline
\end{tabular}

Tabla 5. Valoración de autoestima en pacientes

Sobre autoestima el 33\% refirió sentirse con moral baja lo que revela afectación del, desaliento, y angustia. El 25\% refirieron "siempre" sentirse con baja moral. Mientras las ideas de suicidio revelan que un $77 \%$ nunca haber pensado en suicidarse pero el $23 \%$ que si lo admite es una magnitud elevada y estadísticamente significativa. 


\section{Factores que influyen en la no adherencia al tratamiento antirretroviral en pacientes con VIH/ Sida}

Vol. 2, núm. 1., (2018)

Marjoire Catalina Valle Espin; Freddy Eduardo Villacreces Garcia; Julio Guillermo Chippe

Villacres; Maria Auxiliadora Palma Roditi; Karina Jessenia Jarrin Maisincho

\begin{tabular}{|l|c|c|c|c|c|c|c|c|}
\hline \multirow{2}{*}{ Apoyo familiar } & \multicolumn{2}{|c|}{$\begin{array}{c}\text { Algunas } \\
\text { Seces }\end{array}$} & \multicolumn{3}{|c|}{ Nunca } & \multicolumn{2}{|c|}{ Sin hijos } \\
\cline { 2 - 9 } & $\mathbf{N}^{\circ}$ & $\%$ & $\mathbf{N}^{0}$ & $\%$ & $\mathbf{N}^{\circ}$ & $\%$ & $\mathbf{N}^{0}$ & $\%$ \\
\hline $\begin{array}{l}\text { ¿Es la afectividad } \\
\text { Una rutina en sus } \\
\text { relaciones familiares? }\end{array}$ & 67 & 79 & 16 & 19 & 2 & 2 & & \\
\hline $\begin{array}{l}\text { ¿Recibe apoyo y } \\
\text { Cuidado de su entorno } \\
\text { familiar? }\end{array}$ & 16 & 19 & 29 & 34 & 35 & 41 & 5 & 6 \\
\hline
\end{tabular}

Total de Pacientes 85

Tabla 6. Apoyo del familiar

El 79\% respondió recibir afecto del entorno familiar, pero el $41 \%$ refirió no tener apoyo familiar en formas de cuidado

\section{Consecuencias clínicas relacionadas a la no adherencia}

Las formas de interacción actual entre el servicio de salud de la clínica VIH y el usuario son un factor que no contribuye efectivamente al cumplimento del tratamiento y las indicaciones terapéuticas hecho que se evidencia fundamentalmente en la dimensión intramuros de la terapia que deja el papel activo de cumplimiento al paciente y sus cuidadores, pues el sistema de salud no interviene en el seguimiento comunitario para estimular la decisión de iniciar y mantener el tratamiento antirretroviral El $45 \%$ de los pacientes estudiados presentaron en los controles cifras por debajo de 200 de CD4 y carga viral superior a 50 copias $/ \mathrm{ml}$. 


\section{Factores que influyen en la no adherencia al tratamiento antirretroviral en pacientes con VIH/ Sida}

Vol. 2, núm. 1., (2018)

Marjoire Catalina Valle Espin; Freddy Eduardo Villacreces Garcia; Julio Guillermo Chippe Villacres; Maria Auxiliadora Palma Roditi; Karina Jessenia Jarrin Maisincho

\section{Relación de consecuencias y causalidad social}

El $75 \%$ de los pacientes infectados manifestaron que no ganaban más del sueldo básico y el $25 \%$ no percibe sueldo alguno, esta última población manifestó no contar con los recursos necesarios y económicos para cumplir con los controles y un buen régimen alimenticio. El 58\% manifestó tener la responsabilidad económica sobre hijos y miembros de la familia de cuyo porcentaje abarca el $30 \%$ perteneciente a aquellas pacientes en cuanto al nivel de autoestima el $36 \%$ de pacientes con criterio a la falta de adherencia reveló recibir maltrato físico y discriminación.

\section{Conclusiones.}

Nuestro estudio tuvo como objetivo la exploración de la relación existente entre la adhesión al tratamiento antirretroviral y algunas variables psicológicas y sociales .Se clasificaron los participantes en adherentes y no adherentes por el grado de cumplimiento, analizándose, a continuación, las relaciones que presentaban con algunas variables psicosociales. El énfasis del estudio estuvo dado en los niveles de afectación al autoestima y el apoyo familiar así como a las condiciones socio económicos que afectan el área emocional. Todas estas variables presentan una relación significativa que explicarían el fenómeno de la no adherencia con causas establecidas en el área de la conducta y las emociones las mismas que trastocan con el cumplimiento de la medicación antirretroviral. Con relación a la calidad de vida y algunos factores sociales, se evidenció que el $45 \%$ de pacientes no cumplieron con los parámetros de 


\section{Factores que influyen en la no adherencia al tratamiento antirretroviral en pacientes con VIH/ Sida}

Vol. 2, núm. 1., (2018)

Marjoire Catalina Valle Espin; Freddy Eduardo Villacreces Garcia; Julio Guillermo Chippe Villacres; Maria Auxiliadora Palma Roditi; Karina Jessenia Jarrin Maisincho

adherencia manteniendo una variable en cuanto al inicio, mantenimiento y control de la terapia ARV.

La "feminización" se pone en evidencia en el estudio y está en relación con los datos nacionales e internacionales, La feminización rompe el mito que la enfermedad es exclusiva de prostitutas, la mayoría de las mujeres a infectadas es de ocupación que haceres domésticos

Una conclusión categórica es que la mala adherencia es causa de enfermedades oportunistas.

\section{Bibliografía.}

1. Varela-Arévalo MT, Hoyos-Hernández PA. La adherencia al tratamiento para el VIH/SIDA: más allá de la toma de antirretrovirales. Rev. salud pública. 2015 Sep; 17(4): p. 528-540.

2. OMS. Diez datos sobre el VIH/sida 2011 Ginebra: Organización Mundial de la Salud; 2012.

3. Mellins C, Kang E, Cheng-Shiun L, Havens J, Chesney M. Longitudinal Study of Mental Health and Psychosocial Predictors of Medical Treatment Adherence in Mothers Living with HIV Disease. AIDS Patient Care and STDs. 2003 Oct; 17(8): p. 407-416.

4. Heather RB. HIV-infected childbearing women in Europe: health, treatment and care Londres: UCL Institute of Child Health; 2013.

5. Avery A, Del Toro M, Caron A. Increases in HIV screening in primary care clinics through an electronic reminder: An interrupted time series. BMJ Qual Saf. 2014 Oct; 23(1): p. 250-256.

6. Arrivillaga-Quintero M, Pérez-Flórez M, Borrero-Ramírez YE, Cecilia-Zea M. ¿Qué es primero: desigualdades o inequidades? A propósito del caso de la mortalidad por VIH/ sida en Cali, Colombia (1986-2012). Rev. Gerenc. Polít. Salud. 2016 Feb; 15(31): p. 216-231. 